\title{
MagAO-X first light
}

Males, Jared, Close, Laird, Guyon, Olivier, Hedglen, Alexander, Van Gorkom, Kyle, et al.

Jared R. Males, Laird M. Close, Olivier Guyon, Alexander D. Hedglen, Kyle Van Gorkom, Joseph D. Long, Maggie Kautz, Jennifer Lumbres, Lauren Schatz, Alexander Rodack, Kelsey Miller, David Doelman, Frans Snik, Steven Bos, Justin M. Knight, Katie Morzinski, Victor Gasho, Christoph Keller, Sebastiaan Haffert, Logan Pearce, "MagAO-X first light," Proc. SPIE 11448, Adaptive Optics Systems VII, 114484L (13 December 2020); doi: 10.1117/12.2561682

SPIE. Event: SPIE Astronomical Telescopes + Instrumentation, 2020, Online Only 


\title{
MagAO-X First Light
}

Jared R. Males ${ }^{\mathrm{a}}$, Laird M. Close ${ }^{\mathrm{a}}$, Olivier Guyon ${ }^{\mathrm{a}, \mathrm{b}, \mathrm{c}, \mathrm{d}}$, Alexander D. Hedglen ${ }^{\mathrm{a}, \mathrm{b}}$, Kyle Van Gorkom $^{\mathrm{a}, \mathrm{b}}$, Joseph D. Long ${ }^{\mathrm{a}}$, Maggie Kautz ${ }^{\mathrm{a}, \mathrm{b}}$, Jennifer Lumbres ${ }^{\mathrm{a}, \mathrm{b}}$, Lauren Schatz ${ }^{\mathrm{a}, \mathrm{b}}$, Alex Rodack $^{\mathrm{a}, \mathrm{b}}$, Kelsey Miller ${ }^{\mathrm{e}}$, David Doelman ${ }^{\mathrm{e}}$, Frans Snik ${ }^{\mathrm{e}}$, Justin M. Knight ${ }^{\mathrm{a}, \mathrm{b}}$, Katie Morzinski $^{a}$, Victor Gasho ${ }^{a}$, Christoph Keller ${ }^{\mathrm{e}}$, Sebastiaan Haffert ${ }^{\mathrm{a}}$, and Logan Pearce ${ }^{\mathrm{a}}$

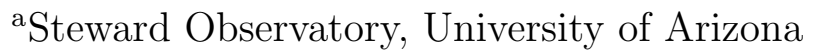

b James C. Wyant College of Optical Sciences, University of Arizona

'Subaru Telescope, National Astronomical Observatory of Japan

dAstrobiology Center, National Institutes of Natural Sciences, Japan

eSterrewacht Leiden, Universiteit Leiden

\begin{abstract}
MagAO-X is a new "extreme" adaptive optics system for the Magellan Clay $6.5 \mathrm{~m}$ telescope which began commissioning in December, 2019. MagAO-X is based around a 2040 actuator deformable mirror, controlled by a pyramid wavefront sensor operating at up to $3.6 \mathrm{kHz}$. When fully optimized, MagAO-X will deliver high Strehls $(>70 \%)$, high resolution (19 mas), and high contrast $\left(<1 \times 10^{-4}\right)$ at $\mathrm{H} \alpha(656 \mathrm{~nm})$. We present a brief review of the instrument design and operations, and then report on the results of the first-light run.
\end{abstract}

Keywords: adaptive optics, wavefront sensing, wavefront control, coronagraphs, high contrast imaging, exoplanets

\section{INTRODUCTION}

MagAO- $\mathrm{X}^{1}$ is a visible-to-near-IR "extreme" adaptive optics (ExAO) system which has begun commissioning on the Magellan Clay $6.5 \mathrm{~m}$ telescope at Las Campanas Observatory (LCO), in Chile. With a 2040 actuator high order deformable mirror (DM) being controlled at up to $3.6 \mathrm{kHz}$ by a pyramid wavefront sensor (PyWFS), MagAO-X is optimized for very high Strehl at short wavelengths. This excellent wavefront quality enables narrowangle high contrast imaging with coronagraphs. Wavefront quality is further augmented using coronagraphic low-order and non-common path wavefront sensing and control (WFS\&C).

The main science goal of the initial operations of MagAO-X is to conduct a survey of nearby $\mathrm{T}$ Tauri and Herbig Ae/Be stars for newly formed accreting planets in $\mathrm{H} \alpha .^{2}$ Using the simultaneous differential imaging (SDI) mode of MagAO-X, this survey will explore the population of low-mass outer extrasolar giant planets (EGPs), revealing where and how such planets form. Additional near-term science cases for MagAO-X include: circumstellar disk characterization; young EGP characterization in the red-optical/near-IR; spatially resolved stellar surface imaging at high spectral resolution; characterization of tight binary star systems; Kepler and TESS followup; and high spatial resolution imaging of asteroid surfaces and asteroid companion searches. The ultimate goal for MagAO-X is the characterization of nearby temperate exoplanets in reflected light. Achieving the demanding wavefront control precision required to perform such observations will require continued improvements and upgrades as MagAO-X commissioning proceeds.

Here we provide a brief overview of the instrument specifications, design, and concept of operations. Following this we report results from our successful first commissioning period. Finally we discuss future plans for MagAO$\mathrm{X}$, including upgrades and use as a laboratory testbed for the Giant Magellan Telescope.

Adaptive Optics Systems VII, edited by Laura Schreiber, Dirk Schmidt, Elise Vernet, Proc. of SPIE Vol. 11448, 114484L · @ 2020 SPIE · CCC code: 0277-786X/20/\$21 · doi: 10.1117/12.2561682 


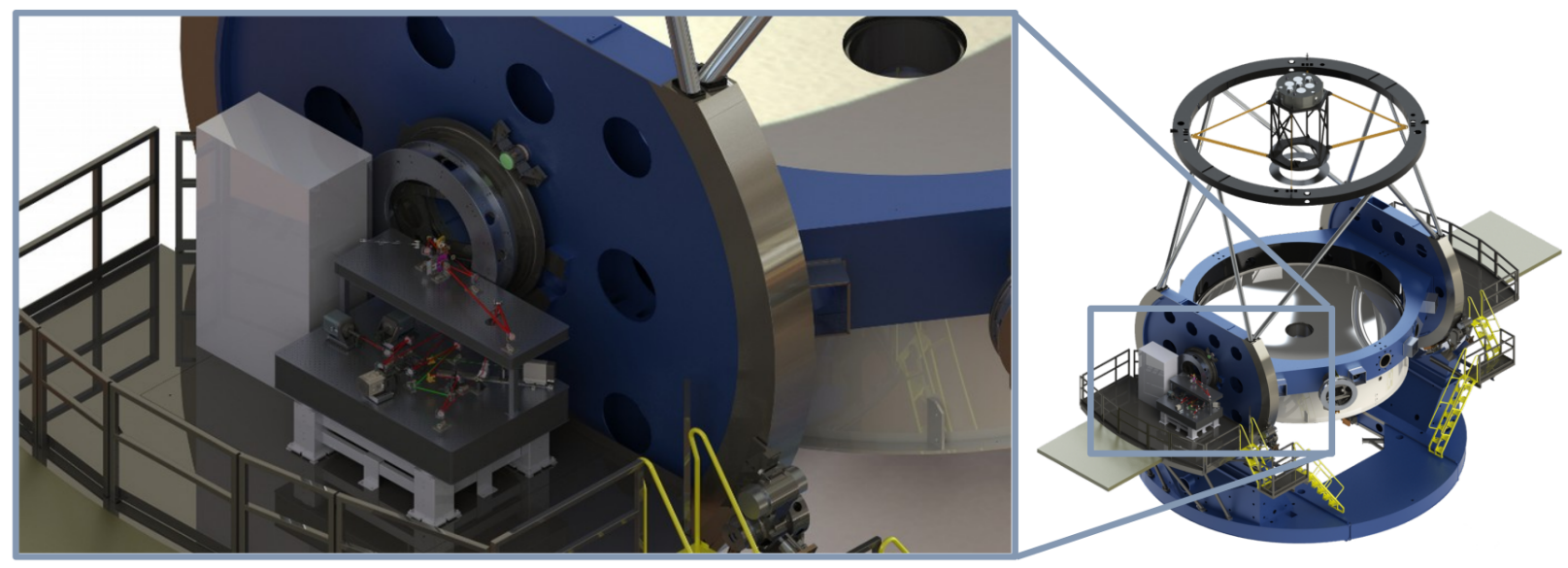

Figure 1. MagAO-X occupies the Nasmyth platform of the Magellan Clay $6.5 \mathrm{~m}$ telescope at Las Campanas Observatory in Chile.

\section{INSTRUMENT OVERVIEW}

\subsection{Design}

Here we provide a very brief overview of the MagAO-X design. See our previous SPIE contributions for more detailed treatments ${ }^{1,3-12}$ In addition, the complete preliminary design review (PDR) documentation is available at https://magao-x.org/docs/handbook/appendices/pdr/, and results of laboratory integration and preparation for shipment can be bound in the pre-ship review (PSR) documentation: https://magao-x.org/docs/ handbook/appendices/psr/index.html

MagAO-X consists of a two-level floating optical table, used to mitigate vibrations, mounted on the gravityinvariant Nasmyth platform of the Magellan Clay 6.5 m telescope at Las Campanas Observatory (LCO), in Chile. See Figures 1 and 2. The top level of MagAO-X contains the de-rotator K-mirror, the atmospheric dispersion corrector (ADC), the woofer DM (an ALPAO DM-97) and the MEMS 2K tweeter. The lower level contains the PyWFS. A beamsplitter sends light to the PyWFS and to the coronagraphic science channel. A selection of dichroic beamsplitters allows optimization of the wavelength split depending on science goals and star brightness.

The coronagraph can be described as a "generalized Lyot" architecture. The first pupil plane houses various masks and apodizers, or in the case of the vector Apodizing Phase Plate (vAPP) the coronagraph itself. A subsequent focal plane holds focal plane masks (FPMs) to block or redirect starlight. These are opaque masks for simple Lyot coronagraphs, and phase shifting complex masks for more advanced designs such as the phase induced amplitude apodization complex mask coronagraph (PIAACMC ${ }^{11}$ ), which is part of the baseline design. The following pupil-plane, usually called the "Lyot plane", contains pupil masks designed to reject residual or redirected starlight.

At either of the FPM or Lyot planes light rejected by the coronagraph is sent to a high speed low noise electron multiplying charge coupled device (EMCCD) camera for low-order and focal-plane wavefront sensing and control (LO\&FP WFS\&C). This allows cleanup of non-common path aberrations, including tracking and controlling temporal evolution, using a third deformable mirror in the coronagraph, which we call the non-common path corrector (NCPC) DM.

The science channel currently consists of a dual-EMCCD simultaneous differential imaging (SDI) system. This allows simultaneous imaging in different photometric bandpasses. Examples include on/off H $\alpha$ (used to detect accretion signatures from proto-planets ${ }^{13,14}$ ), and efficient photometric measurements in, say, i' and z' simultaneously. A selection of dichroic beamsplitters is used to split light depending on the science goals.

Further author information: (Send correspondence to JRM)

JRM: E-mail: jrmales@email.arizona.edu 


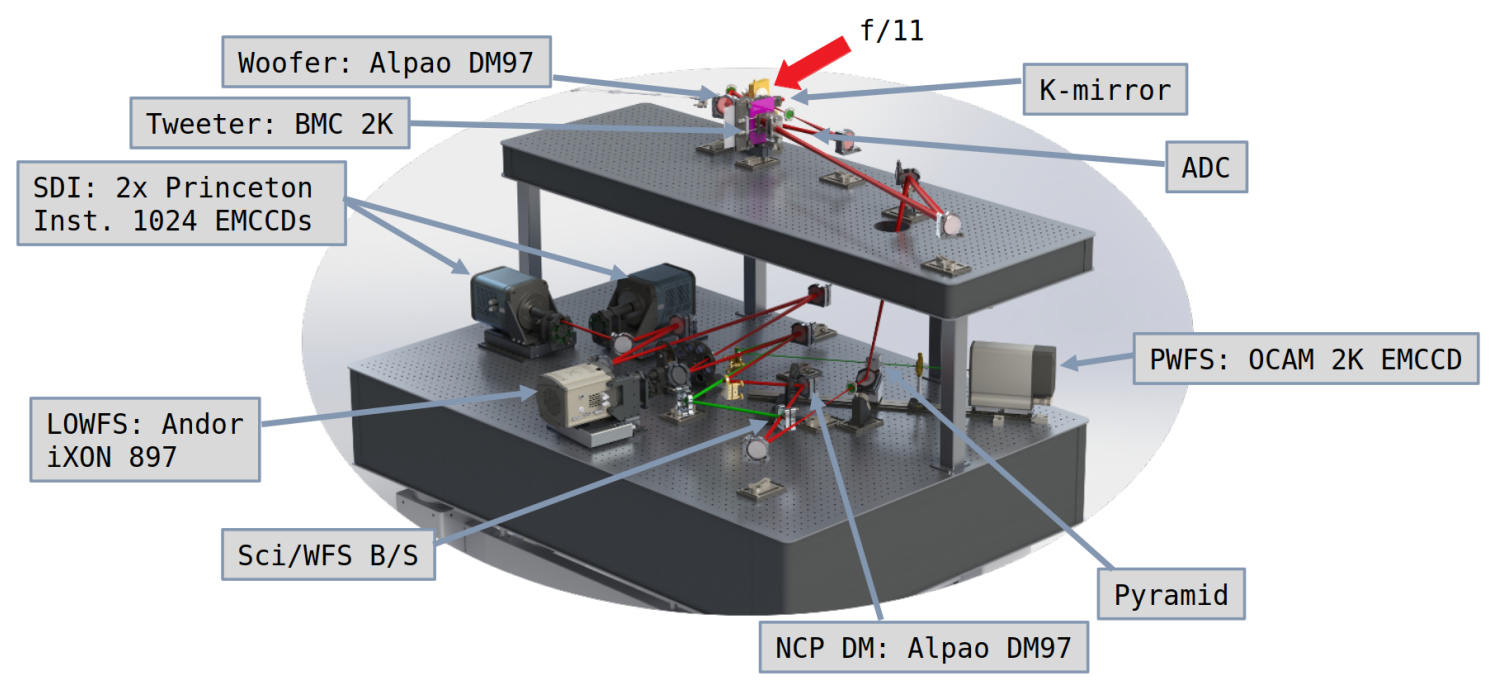

Figure 2. Overview of the MagAO-X opto-mechanical layout. See text for details of the components.

A prototype visible integral field spectrograph (PI: S. Haffert) is currently being tested, and a full version called VIS-X is under development.

The MagAO-X control computers, including the real-time computer, are commercial off-the-shelf multi-core workstations with GPUs on PCIe bus extenders. MagAO-X uses the compute and control for adaptive optics (CACAO) software package for real-time $\mathrm{AO}$ control. ${ }^{12}$ This includes the low-latency interface to all three deformable mirrors via shared memory, as well as the calibration and WFS image processing and reconstruction. Instrument control software is provided by a custom library (https://github.com/magao-x/MagAOX). Non time-critical inter-process communication uses the Instrument Neutral Distributed Interface (INDI). In addition to Qt based GUIs, a javascript browser-based interface is under development.

The instrument user's manual can be viewed at https://magao-x.org/docs/handbook/.

\subsection{Operations}

The MagAO-X project is planned with a philosophy of early deployments for commissioning, followed by laboratory periods of optimization and upgrades. This is to facilitate responding to lessons learned on-sky, and ensure that the algorithms developed for advanced wavefront control are tuned for realistic conditions.

In addition, MagAO-X is a testbed for ExAO development, supporting both laboratory and on-sky testing of new technology. Due to the remote nature of LCO, significant laboratory development and student involvement would not be practicable on site. A carefully engineered shipping system allows us to return the instrument to the lab on Arizona between runs (Figure 3), maximizing access for development and testing. One-way shipping takes approximately two weeks, allowing for efficient transfer between lab and telescope. This enables MagAO-X to serve a broader purpose, for instance as a testbed for the Giant Magellan Telescope. ${ }^{15}$

\section{FIRST LIGHT}

MagAO-X shipped to LCO in Oct, 2019, and saw first light in Dec, 2019. A total of four nights were allocated for installation, alignment, calibration, and initial performance characterization. Fig. 4 shows the instrument on the Clay. Also shown is a z' $(900 \mathrm{~nm}) \mathrm{PSF}$, demonstrating $46 \%$ Strehl ratio (the ratio of peak-height to that of a perfect image, also shown for comparison).

Figure 6 shows the long exposure dark-hole raw contrast behind the vAPP coronagraph ${ }^{16}$ in SDI mode. This observation was in a filter set chosen for SDI using the $875 \mathrm{~nm} \mathrm{CH}_{4}$ feature found in giant planet atmospheres. The intensity in the 2 channels is very similar, as shown by the difference between them. The speckle pattern was essentially constant for the entire observation, showing how stable the opto-mechanics and high-order WFS\&C 

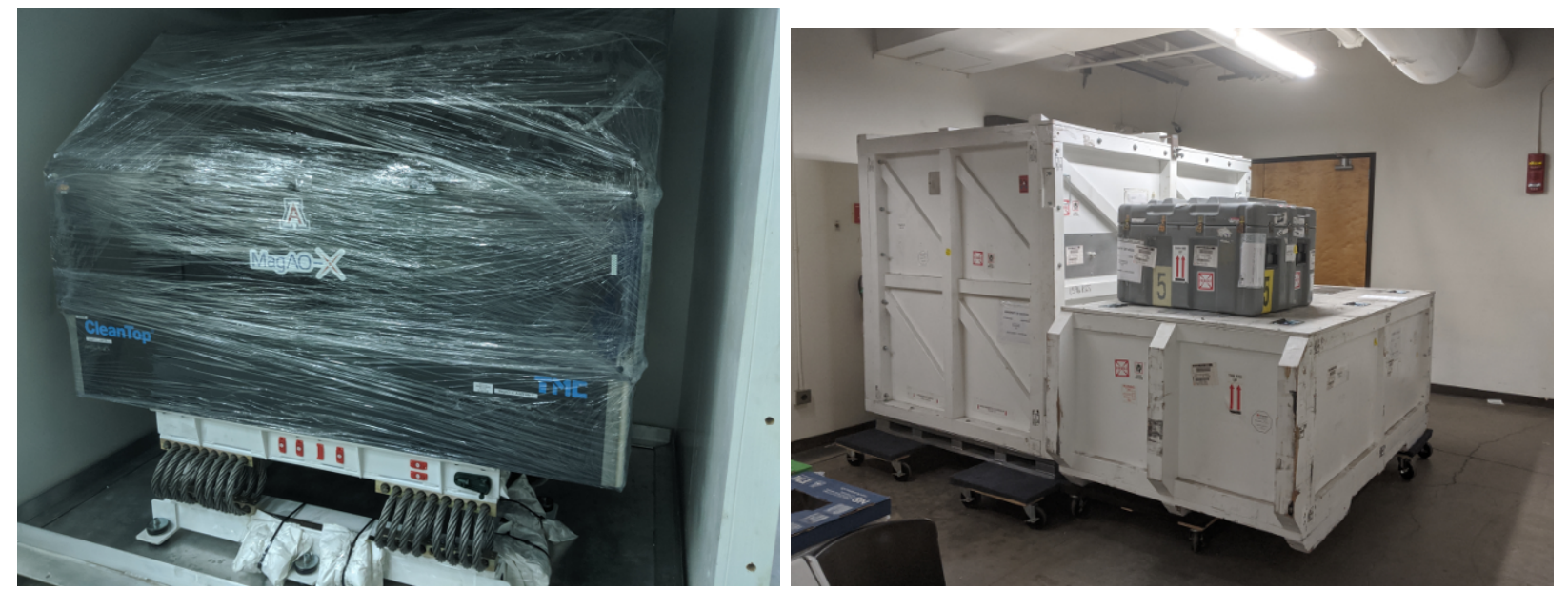

Figure 3. The MagAO-X shipping system. At left, the MagAO-X table assembly sits on the wire-rope isolator frame which safely transports the opto-mechanical system. At right: the entire MagAO-X system, including electronics and operator computer, in the shipping boxes awaiting transport.

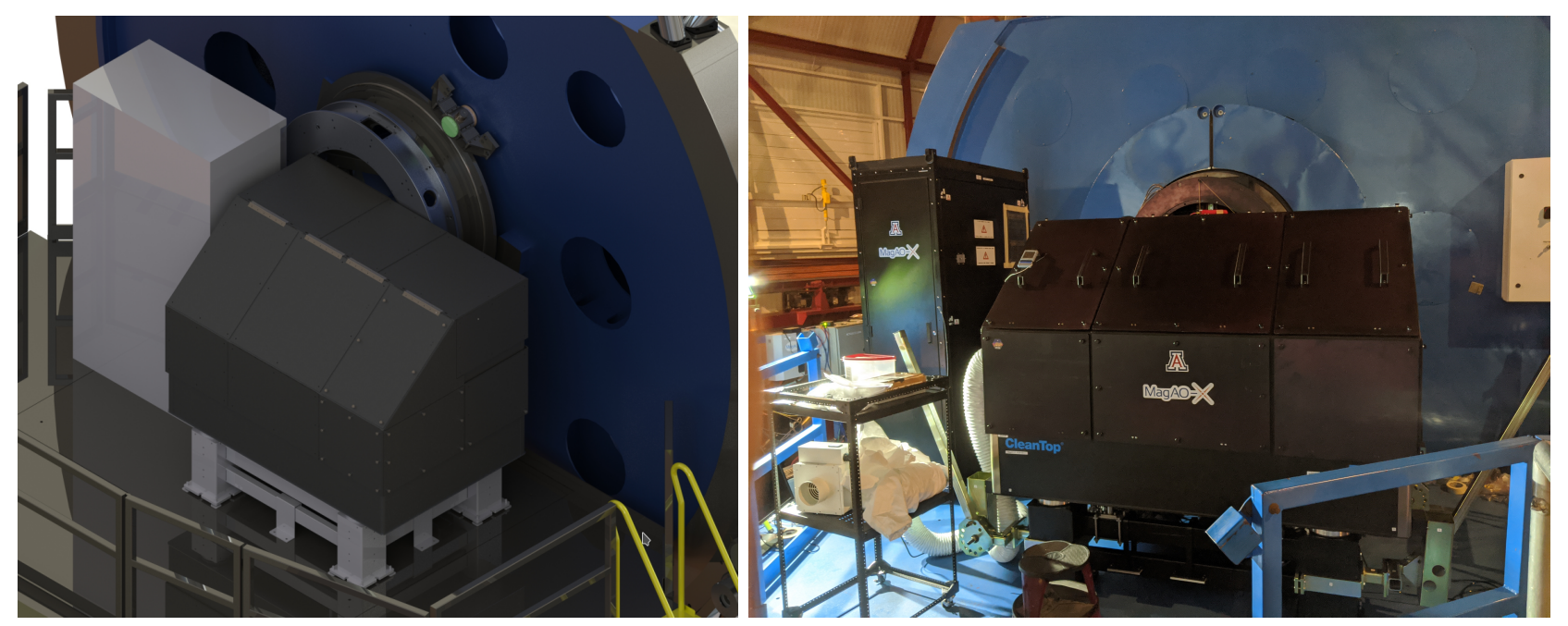

Figure 4. Left: design of MagAO-X on the Clay Nasmyth platform. Same as in Figure 1 but with covers installed for operations. Right: MagAO-X installed at first-light (Dec. 2019). 


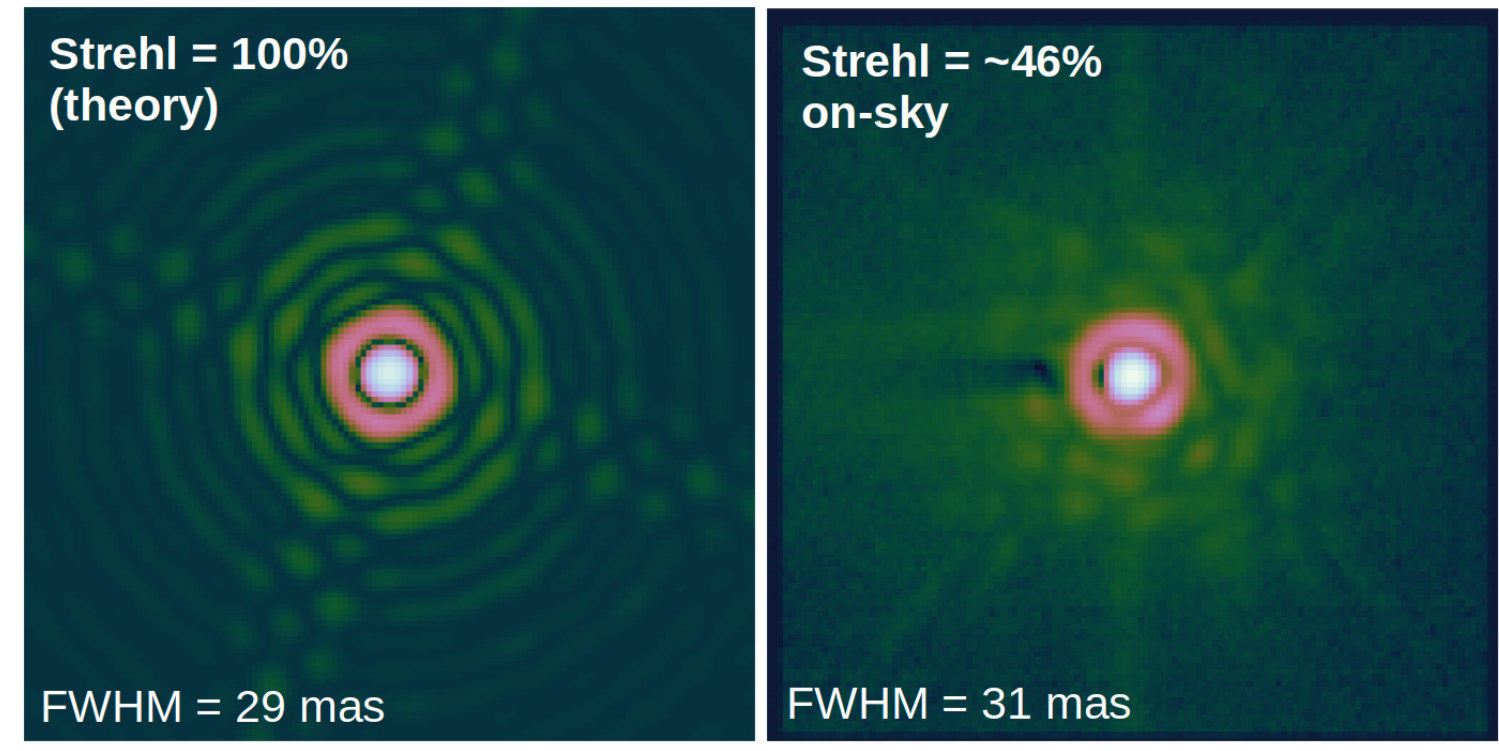

Figure 5. A $z^{\prime}$ PSF measurement taken during commissioning.
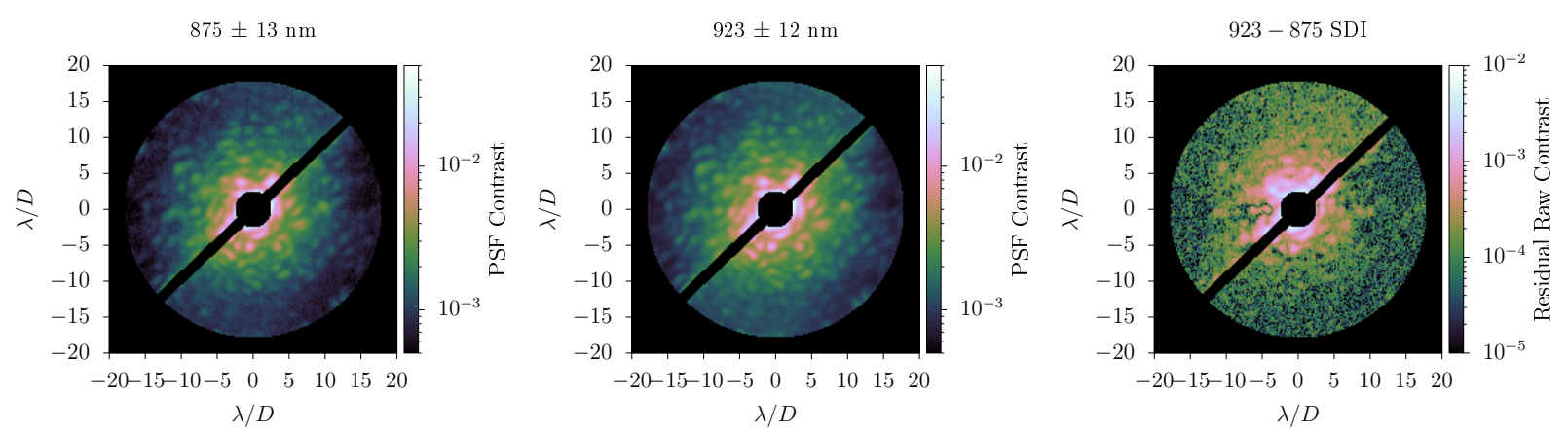

Figure 6. Left: $4 \mathrm{hr}$ average contrast in the $875 \mathrm{~nm}$ filter behind the vAPP coronagraph. Middle: same, but for the 923 nm filter. Right: simultaneous differential imaging (SDI) difference image of the two.

system are. Such highly stable aberrations will be efficiently suppressed using active low-order and non-common path WFS\&C systems being implemented

Fig. 7 shows a calibrated contrast curve from the same $4 \mathrm{hr}$ observation in the $923 \mathrm{~nm}$ filter. The KLIP algorithm ${ }^{17}$ was used for both SDI (using the $875 \mathrm{~nm}$ data as a reference basis) and for ADI on the SDI reduced frames. Throughput was calibrated with simulated planets. The $5 \sigma$ detection contrast was $10^{-4}$ at $0.1 "$.

At the conclusion of the 4 night commissioning allocation, MagAO-X was returned to Tucson to implement fixes for various lessons learned, and for further development and upgrades.

\section{OUTLOOK}

MagAO-X is currently (December 2020) in the lab at UA. Improvements made or in progress as a result of lessons learned on-sky include:

- Flexure between the top and bottom levels, likely due to temperature, resulted in a slight misalignment between the tweeter and PWFS pupils internal to the instrument. This could not be corrected dynamically with the pupil steering mirror. The PWFS pupil imaging lens has now been actuated to allow dynamic realignment in closed-loop. 


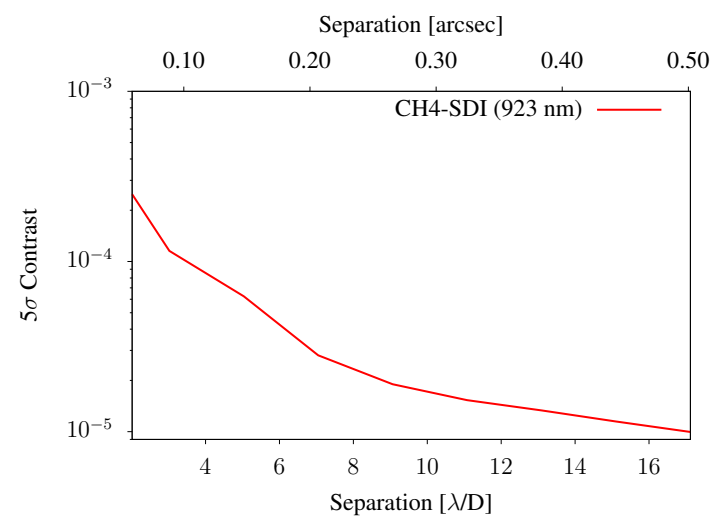

Figure 7. MagAO-X Reduced contrast: results of SDI and ADI processing using the KLIP algorithm, calibrated with simulated planet injection. This performance, particularly at wider separation, shows the excellent stability of MagAO-X. Implementing improvements in both high-order WFS\&C and low-order and focal-plane WFS\&C will deliver over a factor of 100x improvement in detection contrasts.

- The internal telescope simulator illumination pattern was poorly matched to the on-telescope pupil, due to a combination of chromaticity in the simulator optics and beam profile. The result was that high order WFS\&C loop calibration using the simulator did not perform optimally on-sky. The telescope simulator has been upgraded to use a more powerful source, allowing a smaller portion of the fiber output to be used to flatten the profile as well as permitting a narrower band to be use to limit chromaticity.

- Excess vibrations were present. The air-floated actively leveled table did an excellent job rejecting external vibrations, but the positive-pressure blower and the liquid cooling system both added vibrations to the system directly. The blower vibrations are controlled by simply operating at a slower speed during observations. The cooling system vibrations were mitigated by using less stiff hose to couple the pump to the on-table loop, which transmits fewer vibrations.

- The tweeter-to-woofer offload system requires tuning. In on-sky turbulence we noted additional dynamic interaction, which has the effect of increasing apparent servo-lag error. We are implementing a counteroffload system to counter-act this.

Allocations of telescope time in April, 2020 and December, 2020 have been lost due to the SARS-CoV-2 (COVID-19) pandemic. A commissioning run with science verification is tentatively planned for the 2021A semester.

\section{CONCLUSION}

The ExAO system MagAO-X has been integrated and began commissioning at LCO in Dec, 2019. During the first 4-night commissioning run the loop was successfully closed and the vAPP SDI coronagraph was used to demonstrate instrument stability. Additionally various items were identified which are currently being worked in the lab in Tucson to continue progressing towards the aggressive performance goals of the project.

More information about MagAO-X is contained in these proceedings, including: the $H \alpha$ proto-planet survey; ${ }^{18}$ planned spectroscopic upgrades $;^{19}$ the CACAO software package $;{ }^{20}$ a novel wavefront control algorithm being developed; ${ }^{21}$ the use of MagAO-X for GMT testing; $;{ }^{15}$ and pyramid WFS experiments related to MagAO$\mathrm{X} .^{22}$

\section{ACKNOWLEDGMENTS}

We are very grateful for support from the NSF MRI Award \#1625441 (MagAO-X). The research of DD and FS leading to these results has received funding from the European Research Council under ERC Starting Grant agreement 678194 FALCONER). 


\section{REFERENCES}

[1] Males, J. R., Close, L. M., Miller, K., Schatz, L., Doelman, D., Lumbres, J., Snik, F., Rodack, A., Knight, J., Van Gorkom, K., Long, J. D., Hedglen, A., Kautz, M., Jovanovic, N., Morzinski, K., Guyon, O., Douglas, E., Follette, K. B., Lozi, J., Bohlman, C., Durney, O., Gasho, V., Hinz, P., Ireland, M., Jean, M., Keller, C., Kenworthy, M., Mazin, B., Noenickx, J., Alfred, D., Perez, K., Sanchez, A., Sauve, C., Weinberger, A., and Conrad, A., "MagAO-X: project status and first laboratory results," in [Adaptive Optics Systems VI], Society of Photo-Optical Instrumentation Engineers (SPIE) Conference Series 10703, 1070309 (July 2018).

[2] Close, L. M., "The Separation and $\mathrm{H} \alpha$ Contrasts of Massive Accreting Planets in the Gaps of Transitional Disks: Predicted H $\alpha$ Protoplanet Yields for Adaptive Optics Surveys," AJ 160, 221 (Nov. 2020).

[3] Van Gorkom, K., Miller, K. L., Males, J. R., Guyon, O., Rodack, A. T., Lumbres, J., and Knight, J. M., "Characterization of deformable mirrors for the MagAO-X project," in [Adaptive Optics Systems VI], Society of Photo-Optical Instrumentation Engineers (SPIE) Conference Series 10703, 107035A (July 2018).

[4] Hedglen, A. D., Close, L. M., Males, J. R., and Durney, O., "Optical field/pupil rotator with a novel compact K-mirror for MagAO-X," in [Adaptive Optics Systems VI], Society of Photo-Optical Instrumentation Engineers (SPIE) Conference Series 10703, 1070355 (July 2018).

[5] Lumbres, J., Males, J., Douglas, E., Close, L., Guyon, O., Cahoy, K., Carlton, A., Clark, J., Doelman, D., Feinberg, L., Knight, J., Marlow, W., Miller, K., Morzinski, K., Por, E., Rodack, A., Schatz, L., Snik, F., Van Gorkom, K., and Wilby, M., "Modeling coronagraphic extreme wavefront control systems for high contrast imaging in ground and space telescope missions," in [Adaptive Optics Systems VI], Society of Photo-Optical Instrumentation Engineers (SPIE) Conference Series 10703, 107034Z (July 2018).

[6] Close, L. M., Males, J. R., Durney, O., Sauve, C., Kautz, M., Hedglen, A., Schatz, L., Lumbres, J., Miller, K., Van Gorkom, K., Jean, M., and Gasho, V., "Optical and mechanical design of the extreme AO coronagraphic instrument MagAO-X," in [Adaptive Optics Systems VI], Society of Photo-Optical Instrumentation Engineers (SPIE) Conference Series 10703, 107034Y (July 2018).

[7] Kautz, M., Close, L. M., and Males, J. R., "A locking clamp that enables high thermal and vibrational stability for kinematic optical mounts," in [Adaptive Optics Systems VI], Society of Photo-Optical Instrumentation Engineers (SPIE) Conference Series 10703, 107032Q (July 2018).

[8] Rodack, A. T., Males, J. R., Guyon, O., Mazin, B. A., Fitzgerald, M. P., and Mawet, D., "Real-time estimation and correction of quasi-static aberrations in ground-based high contrast imaging systems with high frame-rates," in [Adaptive Optics Systems VI], Society of Photo-Optical Instrumentation Engineers (SPIE) Conference Series 10703, 107032N (July 2018).

[9] Schatz, L. H., Males, J. R., Close, L. M., Durney, O., Guyon, O., Hart, M., Lumbres, J., Miller, K., Knight, J., Rodack, A. T., Long, J. D., Van Gorkom, K., Jean, M., and Kautz, M., "Design of the MagAOX pyramid wavefront sensor," in [Adaptive Optics Systems VI], Society of Photo-Optical Instrumentation Engineers (SPIE) Conference Series 10703, 1070321 (July 2018).

[10] Miller, K., Males, J. R., Guyon, O., Close, L. M., Doelman, D., Snik, F., Por, E., Wilby, M. J., Bohlman, C., Lumbres, J., Van Gorkom, K., Kautz, M., Rodack, A., Knight, J., Jovanovic, N., Morzinski, K., and Schatz, L., "Focal plane wavefront sensing and control strategies for high-contrast imaging on the MagAO-X instrument," in [Adaptive Optics Systems VI], Society of Photo-Optical Instrumentation Engineers (SPIE) Conference Series 10703, 107031T (July 2018).

[11] Knight, J. M., Guyon, O., Lozi, J., Jovanovic, N., and Males, J. R., "Phase-induced amplitude apodization complex-mask coronagraph tolerancing and analysis," in [Advances in Optical and Mechanical Technologies for Telescopes and Instrumentation III], Society of Photo-Optical Instrumentation Engineers (SPIE) Conference Series 10706, 107065 O (July 2018).

[12] Guyon, O., Sevin, A., Gratadour, D., Bernard, J., Ltaief, H., Sukkari, D., Cetre, S., Skaf, N., Lozi, J., Martinache, F., Clergeon, C., Norris, B., Wong, A., and Males, J., "The compute and control for adaptive optics (CACAO) real-time control software package," in [Adaptive Optics Systems VI], Society of PhotoOptical Instrumentation Engineers (SPIE) Conference Series 10703, 107031E (July 2018).

[13] Close, L. M., Follette, K. B., Males, J. R., Puglisi, A., Xompero, M., Apai, D., Najita, J., Weinberger, A. J., Morzinski, K., Rodigas, T. J., Hinz, P., Bailey, V., and Briguglio, R., "Discovery of $\mathrm{H} \alpha$ Emission from the Close Companion inside the Gap of Transitional Disk HD 142527," ApJL 781, L30 (Feb. 2014). 
[14] Wagner, K., Follete, K. B., Close, L. M., Apai, D., Gibbs, A., Keppler, M., Müller, A., Henning, T., Kasper, M., Wu, Y.-L., Long, J., Males, J., Morzinski, K., and McClure, M., "Magellan Adaptive Optics Imaging of PDS 70: Measuring the Mass Accretion Rate of a Young Giant Planet within a Gapped Disk," ApJL 863, L8 (Aug. 2018).

[15] Hedglen, A. et al., "Giant Magellan Telescope high contrast phasing testbed," Proc. SPIE 11448, \#149 (2020).

[16] Otten, G. P. P. L., Snik, F., Kenworthy, M. A., Keller, C. U., Males, J. R., Morzinski, K. M., Close, L. M., Codona, J. L., Hinz, P. M., Hornburg, K. J., Brickson, L. L., and Escuti, M. J., "On-sky Performance Analysis of the Vector Apodizing Phase Plate Coronagraph on MagAO/Clio2," ApJ 834, 175 (Jan. 2017).

[17] Soummer, R., Pueyo, L., and Larkin, J., "Detection and Characterization of Exoplanets and Disks Using Projections on Karhunen-Loève Eigenimages," ApJL 755, L28 (Aug. 2012).

[18] Close, L. M. et al., "Prediction of the planet yield of an AO survey for H-alpha proto-planets with MagAO-X based on first light contrasts," Proc. SPIE 11448, \#28 (2020).

[19] Haffert, S. et al., "Multi-core fibre-fed integral-field unit (MCIFU): Overview and first-light," Proc. SPIE 11448, \#69 (2020).

[20] Guyon, O. et al., "Adaptive optics real-time control with the compute and control for adaptive optics (Cacao) software framework," Proc. SPIE 11448, \#145 (2020).

[21] Rodack, L. et al., "Joint estimation of NCPA and exoplanetary image in stationary atmospheric turbulence using millisecond telemetry from the WFS and science camera," Proc. SPIE 11448, \#137 (2020).

[22] Schatz, L. et al., "First results of laboratory tests of a three-sided pyramid wavefront sensor," Proc. SPIE 11448, \#67 (2020). 